\title{
A Comparative Analysis of Relationship Between Hypothyroidism and Platelet Parameters Including Platelet Indices, Platelet Distribution Width, Plateletcrit and P-LCR
}

\author{
P. Kalaivani Amit kumar ${ }^{1 *}$, Anandhalakshmi Swaminathan ${ }^{2}$, G.Geetha Priya ${ }^{1}$ and Shivashekar Ganapathy ${ }^{1}$ \\ ${ }^{1}$ Department of Pathology, SRM Medical College Hospital and Research Centre, Tamilnadu, India \\ ${ }^{2}$ Department of Physiology, Tagore Medical college, India
}

\begin{abstract}
Background: Patients with hypothyroidism are at increased risk of haemorrhage and there is a tendency to develop thromboembolic complications. MPV which is an indicator of platelet activity can act as an individual independent risk factor for cardiovascular complications. Identification of changes in these parameters using automated cell counters are proved to be cost effective and routinely done during clinical practice. This study is aimed to evaluate the influence of hypothyroidism on platelet indices including platelet count, MPV, PDW, plateletcrit and P-LCR and to compare the results with age matched normal euthyroid subjects.

Methods: Prospective cross-sectional study was conducted for a period of 8 months between 60 hypothyroid subjects and 60 normal adults as a control group. Demographic details were compared between the groups. Fasting blood sample was collected to determine thyroid profile. Complete blood count (CBC) including platelet indices and peripheral smear examination were done.

Results: Male: female ratio was 1:8.6\%, The median age was 43 with a range of 34-52 years. Among the baseline data actual difference was not statistically significant. On comparing with control group T3 and T4 values showed statistically significant decrease ( $p<0.00$ and 0.006 ), and TSH value shows statistically significant increase in $(\mathrm{p}<0.00)$ values. Statistically significant increase was noted in MPV $(p<0.03, t$ value $2.165)$ and platelet distribution width $(\mathrm{p}<0.02, \mathrm{t}$ value 2.334). Significant $\mathrm{p}$ values were not found in platelet count $(\mathrm{p}<0.22, \mathrm{t}$ value 1.238$)$ P-LCR $(\mathrm{p}<0.48$, t value 0.697$)$ and PCT $\mathrm{p}<0.24$, t value 1.188$)$ among the groups.
\end{abstract}

Conclusion: Our results suggest that MPV and PDW are the reliable markers among the platelet parameters, can be used as a CVD risk evaluation parameters in hypothyroid patients.

Keywords: Platelet Parameters, Hypothyroidism, Mean Platelet Volume, Platelet Distribution Width, Cardiovascular Complications

\section{Introduction}

Disorder of thyroid gland either hypo or hyperthyroidism is found to be one of the commonest endocrine disorders identified worldwide. As per the literature data it has been identified that nearly 42 million people in India are victims of these disorders. ${ }^{[1]}$ Hypothyroidism is also prevalent in paediatric age group and in pregnant women. It is evident that women are affected five to six times more frequently than men. ${ }^{[2]}$

Hypothyroidism is defined as a deficiency of the thyroid hormones including triiodothyronine and thyroxin. Hypothyroidism is most common disease of endocrine system which affects all systems including hematopoietic system. A certain extent, normal haematopoiesis especially the coagulation system is influenced by various endocrine organs. Thyroid hormones have an important and influential role on haematopoiesis. The effect of thyroid dysfunction on coagulation cascade is complex and yet to be established. In hypothyroidism, the most common abnormality is anaemia, influence on white blood cells is minimal. ${ }^{[3]}$

Few types of acquired abnormalities of coagulation system have been reported in patients with thyroid functional abnormalities, patients with hypothyroidism are at increased risk of haemorrhage and there is a tendency to develop thromboembolic complications. Long-term follow-up studies have pointed out that there was increased mortality due to cardiovascular and cerebrovascular complications in patients with hyperthyroidism. ${ }^{[3]}$

In case of thyrotoxicosis the important finding is significant thrombocytopenia associated with immune-mediated lytic mechanisms. In hypothyroidism due to decreased production of proteins causes decreased levels of multiple coagulation factors. However, these changes do not cause any significant coagulation manifestations. ${ }^{[4]}$

Searching the recent literature revealed that thyroid dysfunction is related to fibrin formation and fibrinolysis. Few studies proposed that there was an increase in 
fibrinolytic activity in hypothyroidism, some of the recent reports showed the opposite findings. ${ }^{[3,4]}$

Since study on subjects with hypothyroidism showed positive correlation of thyroid hormone status to Mean platelet volume (MPV), it has been postulated in literature that Thyroid stimulating hormone (TSH) can have a prothrombotic effect on platelets. ${ }^{[5]}$ These problems can be understood clearly if we identify actual hematologic abnormalities caused by thyroid hypofunction.

The MPV, PDW (platelet distribution width), PCT (plateletcrit), and P-LCR (platelet large scale ratio) indicate the dimensions of circulating platelet uniformity. Increase in the size of platelets indicates increase in metabolic and enzymatic activity. Mean Platelet volume and PDW are found to be the marker of activated platelets. Conditions such as metabolic syndrome, obesity and hypertension show alterations such as increase in the MPV along with other platelet parameters. These alterations can also be related to increased incidence of venous thromboembolism and vascular disease. It was shown in the previous studies that MPV which is an indicator of platelet activity can act as an individual independent risk factor for cardiovascular complications. ${ }^{[3,5]}$

Identification of changes in these parameters using automated cell counters are proved to be cost effective and routinely ordered by clinicians during clinical practice. These markers are mostly underutilized or ignored though it is easily available. ${ }^{[6]}$

To the best of our knowledge there are few studies available relating hypothyroidism and platelet parameters especially concentrated on platelet parameters such as plateletcrit, P-LCR, MPV, PDW and platelet count. Similar studies with comparative analysis with the healthy controls are still less.

This study is aimed to evaluate the influence of hypothyroidism on platelet indices including platelet count, MPV, PDW, plateletcrit and P-LCR and to compare the results with age matched normal euthyroid subjects.

\section{Materials and Methods:}

Prospective cross-sectional study was conducted in SRM Medical College Hospital and Research centre, Kattankulathur, Tamil Nadu, India. Study was done for a period of 8 months from January 2017 to August 2017, between 60 hypothyroid subjects as a study population and 60 normal adults as a control group.

Institutional scientific and Ethical Committee permission and approval were obtained. Written informed consent was taken from all the study subjects before starting data collection. The results were conveyed and discussed with the concerned treating physician. Information was provided in appropriate regional language.

Demographic details such as socioeconomic status, age and weight were comparable between the test and the control groups. Detailed personal history, treatment history, family history and any significant past illness using a pretested questionnaire were taken. Subjects with history of coagulation disorders, diabetes mellitus, hypertension, peripheral vascular disease, hyper/dyslipidaemia, chronic kidney disease, hepatic disease and those who are on any drugs influencing coagulation were excluded from this present study.

The panel of tests applied are as follows: estimation of blood pressure to rule out hypertension, random blood sugar and lipid profile to rule out diabetes mellitus and hyperlipidaemia respectively. Urine routine including sugar and ketone bodies were also obtained.

Fasting blood sample was collected to exclude diurnal variation. Enzyme Linked Immunosorbent Assay (ELISA) was used to quantitatively determine Triiodothyronine hormone $\left(\mathrm{T}_{3}\right)$, thyroxine hormone $\left(\mathrm{T}_{4}\right)$, and thyroid stimulating hormone (TSH) levels in subjects' serum/ plasma. All patients with established thyroid dysfunction were included in the study and then patients with local or systemic diseases that could affect platelet indices and also with inadequate samples/ samples with clots were excluded from the study. Reference range for thyroid stimulating hormone was $0.03-5.5 \mu \mathrm{IU} / \mathrm{mL}$, and according to this range hypothyroid ( $\mathrm{TSH}>5.5 \mu \mathrm{IU} / \mathrm{mL}$ ) patients were selected for study group. ${ }^{[7]}$

Venous blood sample was collected from all test and control subjects in ethylene diamine tetra acetate (EDTA) coated vacutainer and analysed using automated sevenpart cell analyser Sysmex (XT 1800i) to measure complete blood count (CBC) and platelet indices such as platelet count (PLT), plateletcrit (PCT), MPV, PDW, and plateletlarge cell ratio (P-LCR). Frosted glass slides were stained by Leishman stain as per standard operative protocol and manual microscopic examination of peripheral smears was done for correlation and confirmation of the platelet parameters.

PDW and P-LCR were obtained from a platelet size distribution curve provided by automated analyser. MPV was calculated by the following formula: MPV (fL) = [(plateletcrit (\%)/platelet count $\left(10^{9}\right.$ cells/L) X $\left.\left.10^{5}\right)\right]$. PCT was calculated as the ratio of the platelet volume to the whole blood volume. Platelet distribution width at the 
level of $20 \%$ is considered as PDW. P-LCR was fixed as percentage of platelets with a size of $>12 \mathrm{fL}$. ${ }^{[8]}$

Computerized SPSS 16.0 programmer was used for results entry and calculations. Statistical analysis was done by applying nonparametric tests such as unpaired Student's " $t$ " test and ANOVA. Results were expressed as mean \pm standard deviation (SD) values. The P value of 0.05 or less has been considered as significant.

\section{Results}

The comparison of age, height, weight and body mass index between hypothyroid patients and controls were displayed in Table 1. Male: female ratio was 1:8.6\%). The median age was 43 with a range of 34-52 years. Though there was slight increase of all these parameters in hypothyroid patients the actual difference was not statistically significant in any of the parameters.

Table 2 shows comparison of thyroid profile between test and control groups. On comparing with control group T3 and T4 values showed statistically significant decrease ( $p$ value is 0.00 and 0.006 ), and TSH value shows statistically significant increase in ( $p$ value is 0.00 ) values. The comparison of platelet parameters between test and control groups were shown in Table 3. Statistically significant increase was noted in MPV $(\mathrm{p}<0.03$, $\mathrm{t}$ value 2.165$)$ and platelet distribution width $(\mathrm{p}<0.02, \mathrm{t}$ value 2.334$)$. Significant $\mathrm{p}$ values were not found in platelet count $(\mathrm{p}<0.22$, t value 1.238) P-LCR $(\mathrm{p}<0.48$, t value 0.697$)$ and PCT $\mathrm{p}<0.24$, $\mathrm{t}$ value 1.188) among the groups.

Complete blood count chart from one of the hypothyroid patient is showed in figure 1 , all platelet parameters are increased except platelet count which is low normal in this case. (MPV- 13.3fL, PDW- 19.4fL, P-LCR - 52.8\% and PCT $0.21 \%$ ). Leishman stained peripheral smear examination was done to cross check platelet count and morphology and correlated with the automated cell counts. Figure 2, peripheral smear of hypothyroid patient showed platelet count falling within normal range with occasional small aggregates, however with regard to PDW hypothyroid patients showed few giant platelets exhibiting mild to moderate anisocytosis.

Cumulative platelet histogram from two hypothyroid patients taken in 7 consecutive samples is shown in Figure 3 , there is mild thrombocytopenia and graph extension leading to right side without touching the base correlates with increased MPV and PDW in both cases. The graph also goes upwards and shows second peak indicates increased P-LCR.

Table 1: Comparison of anthropometric data between patients with hypothyroidism and controls.

\begin{tabular}{|c|c|c|c|}
\hline Parameters & $\begin{array}{c}\text { Hypothyroid patients }(\mathbf{n}=\mathbf{6 0}) \\
\text { (Mean } \pm \text { SD) }\end{array}$ & $\begin{array}{c}\text { Controls (n=60) } \\
\text { (Mean } \pm \text { SD) }\end{array}$ & P value \\
\hline Age in years & $43.8 \pm 8.9$ & $38.4 \pm 11.7$ & 0.09 \\
\hline Height in cms & $157 \pm 11$ & $160 \pm 8.8$ & 0.226 \\
\hline Weight in kg & $68 \pm 10.9$ & $63.7 \pm 11.6$ & 0.926 \\
\hline $\begin{array}{c}\text { BMI }{ }^{*} \\
\text { Weight in Kg/ Height }{ }^{2} \text { in meters }\end{array}$ & $27.06 \pm 4.03$ & $23.81 \pm 4.24$ & 0.244 \\
\hline
\end{tabular}

"BMI-Body Mass Index

Table 2: Comparison of thyroid profile values between patients with hypothyroid and control group.

\begin{tabular}{|l|l|l|l|l|}
\hline PARAMETERS & $\begin{array}{l}\text { Controls } \\
\text { MEAN } \pm \text { SD }\end{array}$ & $\begin{array}{l}\text { Hypothyroid } \\
\text { MEAN } \pm \text { SD }\end{array}$ & t value & P value \\
\hline T3 & $3.04 \pm 0.207$ & $1.88 \pm 0.192$ & 9.17 & $\mathbf{0 . 0 0 ^ { * }}$ \\
\hline T4 & $1.3 \pm 0.230$ & $0.9 \pm 0.167$ & 3.49 & $\mathbf{0 . 0 0 6 *}^{*}$ \\
\hline TSH & $2.336 \pm 0.525$ & $8.25 \pm 0.622$ & -17.7 & $\mathbf{0 . 0 0}^{*}$ \\
\hline
\end{tabular}

T3, T4 - Tri \& Tetra iodothyronine, TSH - Thyroid stimulating hormone, * denotes statistically significant $p$ value

Table 3: Comparison of platelet parameters between hypothyroid and controls.

\begin{tabular}{|c|c|c|c|c|}
\hline Platelet parameters & $\begin{array}{c}\text { Hypothyroid patients } \\
(\mathbf{n}=\mathbf{3 0})(\text { Mean } \pm \text { SD) }\end{array}$ & $\begin{array}{c}\text { Controls(n=30) } \\
(\text { Mean } \pm \text { SD) }\end{array}$ & P value & t value \\
\hline PLT & $304.23 \pm 66.15$ & $285.92 \pm 73.81$ & 0.22 & 1.238 \\
\hline PDW & $11.86 \pm 1.77$ & $11.02 \pm 1.27$ & $\mathbf{0 . 0 2}^{*}$ & 2.334 \\
\hline MPV & $10.26 \pm 0.69$ & $9.912 \pm 0.59$ & $\mathbf{0 . 0 3}^{*}$ & 2.165 \\
\hline P.LCR & $25.46 \pm 5.37$ & $24.63 \pm 4.94$ & 0.48 & 0.697 \\
\hline PCT & $0.304 \pm 0.06$ & $0.286 \pm 0.05$ & 0.24 & 1.188 \\
\hline
\end{tabular}

PLT: Platelet count, MPV: Mean platelet volume, PDW: Platelet distribution width, PCT: Plateletcrit, P-LCR: Platelet large cell ratio, * denotes statistically significant $p$ value, 


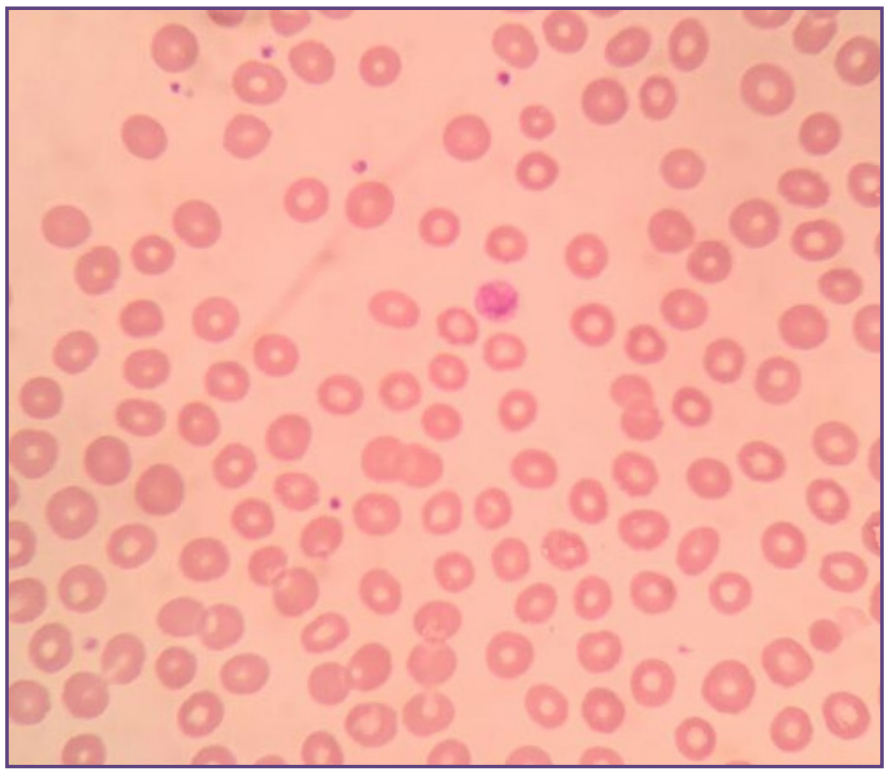

Fig. 1: Peripheral smear in hypothyroid patient.

Peripheral smear, Leishman stain, 1000x, Mild thrombocytopenia (PLT-1,32,000/ cmm) and giant platelet in the centre of the field almost attaining RBC size

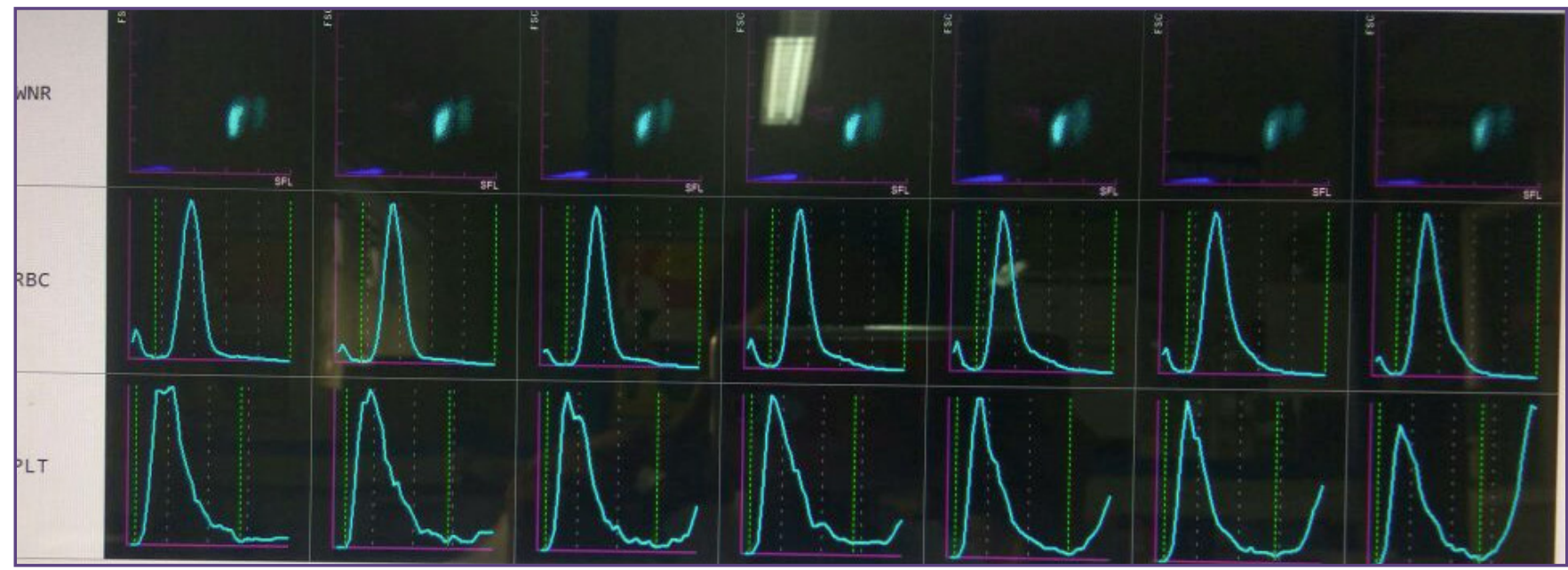

Fig. 2: Cumulative platelet histogram from hypothyroid patients.

Cumulative platelet histogram taken from 7 consecutive samples, show slightly decreased platelet count (115x109/L), and graph extension leading to right side without touching the base correlates with increased MPV (15.3fl) and PDW (19.0fL). The graph also goes upwards and forms second peak indicates increased P-LCR (52.8\%)

\section{Discussion}

The association between thyroid dysfunction and haematological parameter abnormalities and its influence on haematopoiesis are evident from previous observations. Hypothyroidism can cause various forms of anaemia according to the severity and also hyper proliferation of immature erythroid progenitors in few cases. In case of white blood cells and thrombocytes they show slightly depressed counts particularly in hypothyroid patients. It has been also proved that the haematological parameters return to normal level when a euthyroid state is resumed. ${ }^{[9]}$ It is well established fact that platelets play a crucial role in inflammation and coagulation. ${ }^{[10]}$ Hence, evaluation of impact of hypothyroidism on platelet parameters may be helpful in understanding the pathogenesis of coagulation abnormalities or thrombotic events occurring in these patients. Thus, this study is aimed to assess and compare the effects of hypothyroidism on platelet cells count as well as platelet indices including MPV, PDW and other platelet parameters with the healthy euthyroid subjects. 
Sixty patients with hypothyroidism and 60 healthy subjects with euthyroid state were matched for age and body mass index and enrolled for this current study. None of the study subjects had hyper/dyslipidaemia, diabetes mellitus, hypertension or with the history of previous cardiovascular illness or coagulation abnormalities. All the study subjects were evaluated for biochemical and platelet parameters.

Our results with respect to platelet count is correlating with the study conducted by Dorgalalaleh et al who have analyzed the effects of thyroid dysfunctions on blood cell count and red blood cell indices and showed statistically significant differences between hyper, hypothyroid and control groups in RBC count, RBC indices, RDW, Hemoglobin and Hematocrit values $(\mathrm{P}$-value $<0.05$ ), but the difference was not statistically significant for WBC counts and platelet counts (P-value $>0.05)$. ${ }^{[7]}$ According to the obtained data, MCV was statistically different between patients with hypothyroidism and control group (P-value $<0.03$ ). PDW also showed statistically significant difference between control and test group with the $p$ value of 0.02 , but platelet count, P-LCR and plateletcrit did not show statistically significant differences in these two groups of patients (P-value $>0.05$ ).

Recently the prognostic value of platelet indices is being evaluated by many authors, concentrating mainly on MPV in both haematological and non-haematological disorders. The results vary significantly from each other. In a study done by Jung et al in cardiovascular disease patients, it was obseved that MPV levels were high in patients who also had coronary artery calcification, hence they proved that MPV was an independent prognostic factor for cardiovascular mortality. ${ }^{[1]}$

In a seven years study done by Slavka et al, patients who showed increased MPV levels ( $\geq 11.01 \mathrm{fL}$ ) were seen with increased risks of mortality due to ischemic heart disease. The authors discussed that increase in MPV levels would have contributed to the risk identified and they proposed few mechanisms behind these effects such as larger platelets were more active and had more alpha granules which can induce blood vessel intima proliferation than smaller platelets due to increased metabolic and enzymatic activity. As it contains prothrombotic activators like thromboxane A2 and also having procoagulant surface proteins it acts in enhancing thrombus formation which might lead to myocardial infarction, they concluded that MPV was a simple and reliable marker which can assess functional activity of the platelet and its effects on blood vessels. ${ }^{[12]}$

Our study results are concordant with Erick at el ${ }^{[13]}$ who conducted a study including forty-seven patients with subclinical hypothyroidism and compared the results with 30 euthyroid control group and showed that test population had increased mean platelet volume (MPV) and platelet distribution width (PDW) values than normal healthy control group, which were statistically significant and concluded that MPV and PDW values have played significant predictive role in case of subclinical hypothyroidism. Activation of platelets produces larger as well as irregular platelets with plenty of pseudopodia formation in turn causes variation of size and shape and increased anisopoikilocytosis which contributes to increase PDW in case of hypothyroidism.

Plateletcrit indicates platelet mass index measured by calculating platelet count and MPV values. Recently, PCT is being utilized to decide about the need of platelet transfusion. Few studies have showed that platelet parameters including PCT can be used as markers of inflammation in cancer patients. In a study done by Dincel $\mathrm{O}$ et al., PCT was found to be significantly higher in patients with malignant thyroid tumours than multinodular goitre patients. ${ }^{[14]}$ Another study done by Oncel $\mathrm{M}$ et al also proposed that platelet indices would be useful to identify and to follow-up cardio and cerebrovascular, thromboembolic as well as inflammatory diseases. ${ }^{[15]}$ In our study, PCT values were slightly higher in test group than control group, however, no statistically significant difference was identified between the groups. Our study results are correlating with Erdal Uysal et al's study, a retrospective observational study included 389 patients who underwent total thyroidectomy. ${ }^{[16]}$

Francesco Scavuzzo et al studied evaluation of platelet indicators in individuals affected by subclinical and clinical (overt) hypothyroidism. Their study showed that the PCT and $\mathrm{p}$-LCR remained unaltered in overt hypothyroidism or sub-clinical hypothyroidism and MPV and PDW were found to be significantly modified in both groups thus concluded a hypothetical increase of thromboembolic risk in those patients. ${ }^{[5]}$ Our study has showed slight increase in the P-LCR value in hypothyroid patients in comparison with controls, however the difference was not statistically significant. Further large studies are necessary to identify the exact role of PCT and P-LCR in hypothyroid patients.

In the initiation and evolution of atherosclerosis and cardiovascular disease, endothelial dysfunction is found to be a significant factor which causes platelet aggregation. Few novel inflammatory markers have been proposed by recent studies are costly and involved complexity in processing techniques. ${ }^{[17]}$ Hence, easily measurable platelet parameters can be used as markers of endothelial functional derangement and for Cardiovascular disease (CVD) risk assessment. 


\section{Conclusion}

In our study, we showed statistically significant increase in MPV and PDW in hypothyroid patients on comparison with healthy euthyroid subjects. However, there is no significant difference found in the platelet count, plateletcrit and P-LCR between the groups. Measurement of these platelet parameters are affordable and routinely available in most of the laboratories, can be used as a predictive marker to identify hypothyroid patients who are at increased risk of atherothrombotic complications in future. Our results suggest that MPV and PDW are the reliable markers among the platelet parameters, thus can be used as a CVD risk evaluation parameters in hypothyroid patients.

\section{References}

1. Unnikrishnan A.G, Menon U.V. Thyroid disorders in India: An epidemiological perspective. Indian J. Endocrinol. Metabol., 2011;15:78-81.

2. Shah S.N., Joshi S.R. Thyroid as an endocrine organ. JAPI 2000; 48: 7-8.

3. Monika H, Alois G, Arnulf F et.al. Altered Platelet Plug Formation in Hyperthyroidism and Hypothyroidism. The Journal of Clinical Endocrinology \& Metabolism 2007; 92: 3006-3012

4. Singh V, Catlett, Joseph P. Hematologic Manifestations of Thyroid Disease. The Endocrinologist. 1998; 8: 2

5. Francesco S, Maria L G, Gianluca N, et al. Hypothyroidism and platelet parameters evaluation: a preliminary study. Endocrine Abstracts 2015; 37: 965

6. Chu SG, Becker RC, Berger PB, Bhatt DL, Eikelboom JW, Konkle B, et al. Mean platelet volume as a predictor of cardiovascular risk: A systematic review and meta-analysis. J ThrombHaemost. 2010; 8:148-56.

7. Dorgalale A, Mahmoodi M, Varmaghani B. Effect of Thyroid Dysfunctions on Blood Cell Count and Red Blood Cell Indices Iran J Ped Hematol Oncol. 2013; 3(2): 73-77.
8. Ren et al. No associations exist between mean platelet volume or platelet distribution width and thyroid function in Chinese. Medicine 2016; 95:40

9. Iddha M.A, Macharia B.N, Ng'wena A.G. Thryroid Hormones and Hematological Indices Levels in Thyroid Disorders Patients at Moi Teaching and Referral Hospital. ISRN Endocrinology. 2013;1-10

10. Sahbaz A, Cicekler H, Aynioglu O, Isik H, Ozmen U. Comparison of the predictive value of plateletcrit with various other blood parameters in gestational diabetes development. J ObstetGynaecol. 2016; 36(5):589-93.

11. Jung DH, Lee HR, Lee YJ, et al. The association between coronary artery calcification and mean platelet volume in the general population. Platelets 2011; 22: 567

12. Slavka G, Perkmann T, Haslacher H, et al. Mean platelet volume may represent a predictive parameter for overall vascular mortality and ischemic heart disease. Arterioscler Thromb Vasc Biol. 2011;31: 1215-8.

13. Erikci AA, Karagoz B, Ozturk A et al. The effect of subclinical hypothyroidism on platelet parameters. Hematology 2009;14(2):115-7.

14. Dincel O, Bayraktar C. Evaluation of platelet indices as a useful marker in papillary thyroid carcinoma. Bratisl Lek Listy. 2017;118(3):153-5.

15. Oncel M, Kiyici A, Oncel M, Sunam GS, Sahin E, Adam B. Evaluation of Platelet Indices in Lung Cancer Patients. Asian Pac J Cancer Prev. 2015;16(17):7599-602.

16. Erdal U, Seyit M C, Efe S et al. Evaluation of Hemocytometer Parameters as Potential Biomarkers in Benign Multinodular Goiter and Papillary Thyroid Carcinoma. Iranian Red Crescent Medical Journal. 2017; 19: 12

17. Yeom E, Byeon H, Lee SJ. Effect of diabetic duration on hemorheological properties and platelet aggregation in streptozotocin-induced diabetic rats. Sci Rep. 2016; 6:21913.

*Corresponding author:

Dr. P. Kalaivani M.D., Postal Address: S1, Thanya regency, 11/61 Ganesh nagar main road, Selaiyur, East Tambaram, Chennai - 600073 India

Phone: +91 9843575500

Email: drkalaivani1980@gmail.com

Financial or other Competing Interests: None. 